\title{
Infrared Thermographic Analysis of Craniofacial Muscles in Military Pilots Affected by Bruxism
}

\author{
Alberto Baldini; Alessandro Nota; Clementina Cioffi; Fabiana Ballanti; Paola Cozza
}

\begin{abstract}
INTRODUCTION: Due to the physical stresses to which they are subjected, military pilots may experience bruxism, an "oral parafunction." Parafunction can cause masticatory muscle suffering and serious dental, periodontal, and temporomandibular joint damage. The aim of this pilot study was to analyze the temperature distribution in masticatory and upper trapezius muscles in a sample of bruxist air force pilots, to evaluate whether an occlusal splint would be able to induce skin temperature variations in the stomatognathic apparatus using the technology of infrared thermography.

METHODS: A total of 11 male Italian Air Force pilots of high performance aircraft, ages from 27 to $40 \mathrm{yr}$ (mean $34.91 \pm 2.15 \mathrm{yr}$ ) with 1000-3000 flight hours, were enrolled in the study and analyzed using an infrared camera in order to evaluate the temperature of the masticatory muscles. All the recordings were taken on each subject using the same protocol with and without a temporary occlusal splint.

RESULTS: The occlusal splint statistically increased each muscle temperature $\left(0.10-0.20^{\circ} \mathrm{C}\right)$ on both the sides of the body. No statistically significant differences were found between the left and right muscles (asymmetries) before or after the wearing of the splint except for the anterior temporalis muscle. No significant improvement or variations in temperature symmetry of this muscle was found after the application of the splint.

DIScussion: The use of an occlusal splint could help in increasing muscles temperatures in Air Force pilots with consequent relaxation of their facial muscular system.

KEYWORDS: dental occlusion, temporomandibular disorders, occlusal splint, military personnel, muscular tension.
\end{abstract}

Baldini A, Nota A, Cioffi C, Ballanti F, Cozza P. Infrared thermographic analysis of craniofacial muscles in military pilots affected by bruxism. Aerosp Med Hum Perform. 2015; 86(4):374-378.

A ir force aerobatic flight exposes pilots to accelerative stress. ${ }^{3,6}$ Air force pilots' experiences are similar to those of high-performance athletes: both are stressed by accelerations. ${ }^{1}$ Acceleration is the vectorial physical quantity that describes the variation of velocity in a unit of time: $a=d v / d t$. Modern military airplanes are capable of initiating and sustaining instantaneous acceleration forces up to $+9 \mathrm{G}_{\mathrm{z}}$ (gravitational forces). The physiological and operational impact of acute exposures to high-G acceleration has been well documented. ${ }^{20}$

Pathologies concerning dental and stomatognathic problems affecting pilots have been reported in literature. Barodontalgia, the dental pathology that is diagnosed with the highest frequency, occurs in $11 \%$ of air force aircrews at a rate of 5 episodes/1000 flights. ${ }^{23}$ Another pathology, frequently reported during the WWII period, deals with fracturing of restorations during high-altitude flying and is called "dental barotrauma." 24
It is generally accepted that chronic stressful situations and mental diseases contribute to the development of occlusal parafunctions and temporomandibular disorders without being the only cause. ${ }^{22}$ Air force pilots, due to the physical stresses to which they are subjected, can experience bruxism, an oral parafunction that can cause masticatory muscle pain, serious dental and periodontal damage, and temporomandibular joint disorders (TMD). ${ }^{6}$

TMD is a multifactorial pathology, difficult to diagnose and characterized by an alteration of the masticatory muscles,

\footnotetext{
From the University of Rome Tor Vergata, Rome, Italy, and the Italian Air Force Institute of Improvement and Training in Aerospace Medicine, Rome, Italy.

This manuscript was received for review in July 2014. It was accepted for publication in November 2014

Address correspondence to: Dr. Alberto Baldini, Via S. Orsola 5, Bergamo 24122, Italy; studiomedicobaldini@gmail.com.

Reprint \& Copyright $(\odot$ by the Aerospace Medical Association, Alexandria, VA.

DOI: $10.3357 / \mathrm{amhp} .4115 .2015$
} 
limitation of jaw movement, pain, joint noises, and other symptoms. ${ }^{4,8,19}$ In 2007, Lurie et al. performed a study in which a significant percentage (69\%) of Israeli air force pilots analyzed were affected by bruxism; the authors suggested the utility of a protective treatment for the teeth. ${ }^{14}$

In air force pilots, it is important to evaluate various aspects of the masticatory muscles, such as temperature, due to the high stress which this component of the stomatognathic system is subjected to. Stomatognathic diseases, such as myogenous TMD and bruxism, can alter the temperature of masticatory muscles ${ }^{7}$ because it is dependent not only on energy production, but also blood flow. TMD and bruxism are characterized by hyperactivity of the masticatory muscles, which changes the microcirculatory dynamics due to compression of blood vessels, resulting in a decrease of skin temperature. ${ }^{18}$ Thus, in the masticatory system, lower temperatures are typical of muscular hyperactivity while higher temperatures are related to muscular relaxation.

To measure peripheral temperature, infrared thermography is applied ${ }^{9,12,13}$ and is able to detect variations of skin temperature, showing changes in the muscular activity of the masticatory system. Thermography analyzes body infrared radiation with a temperature above absolute zero and is used as an effective tool for the diagnosis of various diseases given its efficiency in analyzing the distribution of skin surface temperature. ${ }^{17}$ Control of heat transfer with the environment plays a fundamental role for body temperature regulation; indeed, the skin temperature distribution of the human body depends on the complex relationships defining the heat exchange processes between skin tissue, inner tissue, local vasculature, and metabolic activity. ${ }^{16}$ Some great advantages of infrared thermography are that it is not an invasive exam, but a "touchless" and dynamic imaging investigation technique. Some studies have been carried out with infrared thermography to measure the skin surface temperature of the masticatory muscles and temporomandibular joint in individuals with TMD. ${ }^{7,8,10}$

It is well established in the field of thermography that healthy, symptom-free patients show thermal symmetry between the right and the left sides of the body. Studies have shown that in healthy persons, temperatures of the body (including the face) were essentially bilaterally equal, with an average temperature difference from side to side (termed AT) of only $0.2^{\circ} \mathrm{C}$. $^{21}$ There are conflicting reports regarding the trustworthiness of this instrument in the diagnosis of TMD because of the difficulty matching its results with the results of the Research Diagnostic Criteria for Temporomandibular Disorders system. ${ }^{18}$ The aim of this pilot study was to analyze the temperature distribution in the masticatory and upper trapezius muscles in a sample of air force pilots, to evaluate whether an occlusal splint was able to induce skin temperature variations in the stomatognathic apparatus.

\section{METHODS}

\section{Subjects}

A total of 11 male Italian Air Force pilots of high performance aircraft, ages from 27 to $40 \mathrm{yr}$ (mean $34.91 \pm 2.15 \mathrm{yr}$ ) with
1000-3000 flight hours, were enrolled in the study and analyzed at the Institutes of Aerospace Medicine in Milan. These pilots were all members of the P.A.N. (National Acrobatic Patrol) and they signed a consent form after being fully informed about the nature of the study. A clinical examination and anamnesis showed all pilots were affected by bruxism, but with no symptoms of TMD (Fig. 1).

\section{Equipment}

An infrared camera FLIR Systems ${ }^{\circledR}$ (Wilsonville, OR) ThermaCAM SC640 with resolution of $640 \times 480$ pixels was used in order to evaluate the temperature of the right and left masseter, right and left anterior temporalis, right and left upper trapezius, and right and left sternocleidomastoid muscles. The instrument was stabilized for 10 min before the exam.

\section{Procedure}

The study protocol was approved by the relevant ethical committee of the Italian Air Force's Forensic Medicine Medical Commission.The participants were asked to avoid hot showers or baths, topical agents, creams or powders, or nasal decongestants, vigorous exercise, or intake of stimulants such as caffeine $2 \mathrm{~h}$ before data collection. During the examination, the pilots stayed in an acclimatized room for $20 \mathrm{~min}$ with a temperature of $22 \pm 0.1^{\circ} \mathrm{C}$, an aeration of $0.3 \pm 0.2 \mathrm{~m} \cdot \mathrm{s}^{-1}$, and a relative humidity of $48 \pm 1 \%$. The subjects were positioned upright without shoes on a posture scale analyzer; the head was positioned with the Frankfurt plane parallel to the ground at a distance from the front lens of the camera of $2 \pm 0.1 \mathrm{~m}$. The region of the muscles analyzed was cleared of clothes and personal items.

In order to evaluate the temperature we used the line tool of the software. This instrument delimits the entire length of the muscles we wanted to study and generates a thermic plan (Fig. 2). For the masseter muscle, the starting point of the line tool was positioned in the zygomatic arch and the final part in the lateral surface of the angle of mandible; for the anterior temporalis muscle, the initial point of the line tool was placed on the frontal bone, immediately above the muscle belly, and the final portion near the eyelid lateral commissure; for the upper trapezius muscle, the starting part of the line tool was positioned on the $\mathrm{C} 7$ spinous process and the final part on the

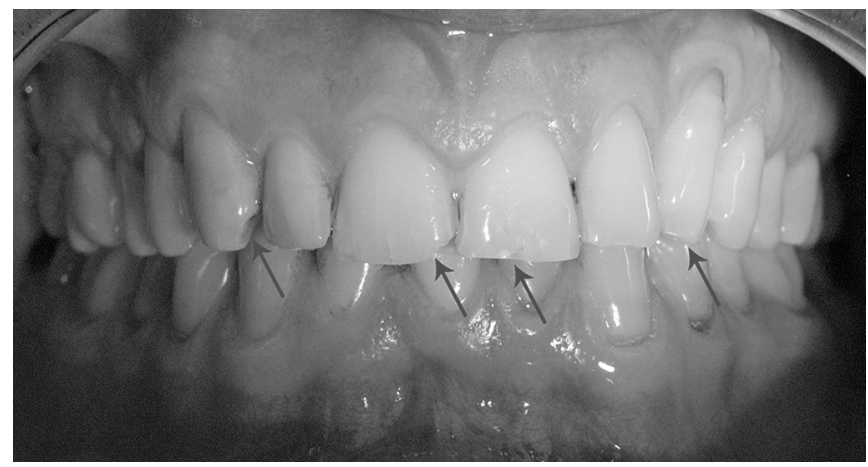

Fig. 1. Clinical intraoral image showing signs of bruxism in an air force pilot involved in the study. 


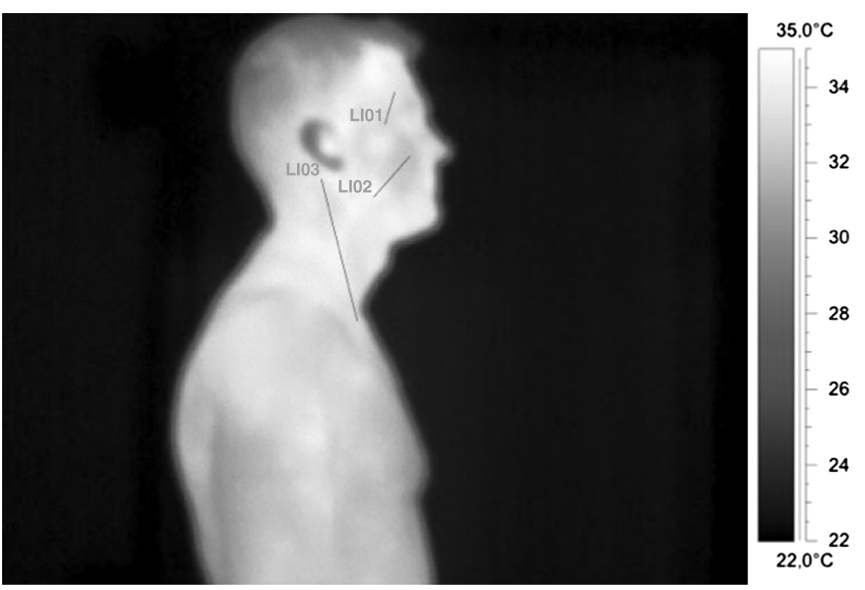

Fig. 2. Example of thermographic analysis of the craniofacial muscles (see the online figure for color)

acromion; for the sternocleidomastoid the initial part of the line tool was positioned on the mastoid process and the final part near the collarbone. The average temperature of the entire line extension was generated along the entire length of the delimited muscle. In order to exclude potential interrater variability, the same operator performed all analyses.

A temporary occlusal splint was fabricated for each subject using Easybite ${ }^{\circledR}$ (Techim Group S.r.l., Milan, Italy). Easybite ${ }^{\circledR}$ consists of a PVC tube, shaped to adapt to the dental arch, that can be filled with a photopolymerizable fluid (Fig. 3 and Fig. 4). An expert operator inserts the Easybite ${ }^{\circledR}$ into the subject's mouth and adds the fluid in order to obtain the ideal shape while asking the patient to bite lightly the tube. In this study the Easybite ${ }^{\circledR}$ was used in a centric relation with Dawson's bilateral manipulation, which is a manual technique that allows the gnathologist to position the mandible and its condyles in a recordable and modifiable position. Studies in literature concluded this technique to be trustworthy and reliable for recording the patient's centric relation position. ${ }^{3}$

The operator stands behind the patient and places three fingers (first finger, middle finger, and ring finger) under the horizontal branch of the mandible with the thumb firmly

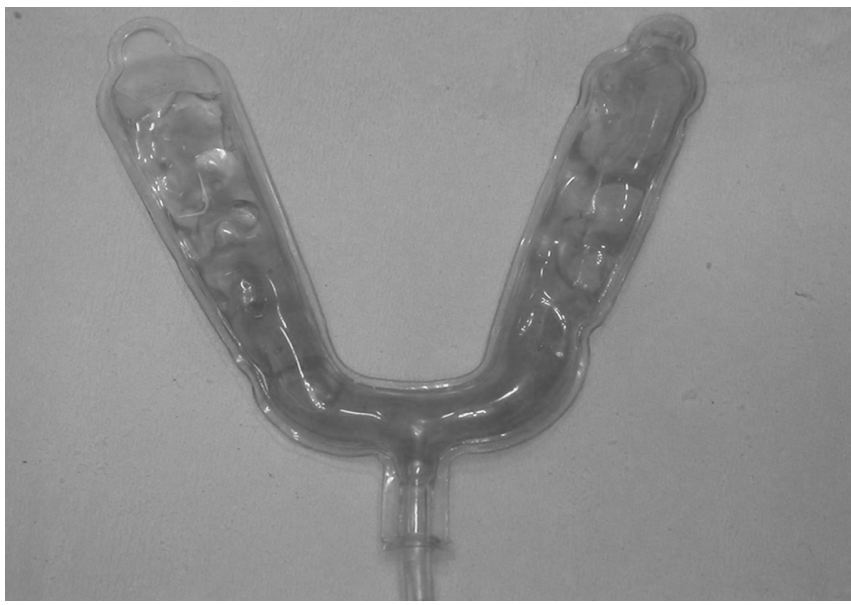

Fig. 3. Filling of an Easybite ${ }^{\circledR}$ with photopolymerizable fluid. positioned on the chin and the little finger on the rear of the vertical branch of the mandible so the operator can pull the mandible, allowing the condyles to return to the glenoid cavity. ${ }^{6}$ Once the operator has obtained the bite in centric relation, the fluid can be photopolymerized.

All of the recordings were taken on each subject using the same protocol with and without the Easybite ${ }^{\circledR}$ with a pause of $2 \mathrm{~h}$ between the recordings. The pilot was asked to have the Easybite ${ }^{\circledR}$ between his dental arches during 20 min of staying in the acclimatized room before the recording. A Student's $t$-test was applied for analyzing the differences between the recordings (statistical significance was set at $P<0.05$ ).

\section{RESULTS}

Table I displays the mean temperature of the muscles on the right and left sides as affected by the occlusal splint. Analyzing the results obtained, it was observed that wearing the occlusal splint statistically increased each muscle temperature on both sides $(P<0.01)$ (Table I). No statistically significant differences were found between the left and right muscles (asymmetries) before or after the wearing the splint except for the anterior temporalis muscle ( $P=0.019$ before and $P=0.023$ after). No significant improvement or variations in temperature symmetry of this muscle was found after the application of the splint.

\section{DISCUSSION}

Some authors have stated that thermography is a record of the heat produced by facial vessels and by the cellular metabolism during muscular activity. $8,16,21$ Thermography was used in some studies in the dental field in order to analyze its usefulness in the analysis of TMD and stomatognathic diseases with contrasting results about the accuracy in diagnosis. ${ }^{18}$ Stomatognathic system diseases such as TMD, inflammations, or muscle and ligament injuries can alter the temperature of the compromised structures and these variations can be detected by telethermographic analysis. Also, bruxism can alter the craniofacial muscles' temperature owing to the consequent hyperactivity.

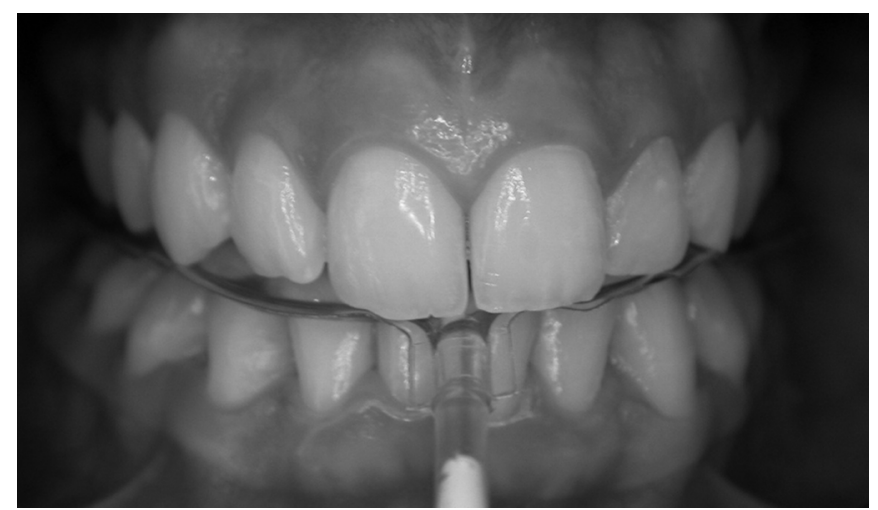

Fig. 4. Easybite ${ }^{\circledR}$ positioning between the dental arches. 
Table I. Differences in Temperature Recorded With and Without the Occlusal Splint.

\begin{tabular}{|c|c|c|c|c|c|c|}
\hline & \multicolumn{2}{|c|}{ MEAN TEMPERATURE ( $\left.{ }^{\circ} \mathrm{C}\right)$} & \multicolumn{4}{|c|}{ STUDENT'S $t$-TEST } \\
\hline & WITHOUT SPLINT & WITH SPLINT & DIFF & D.F. & $t$ & P-VALUE \\
\hline \multicolumn{7}{|c|}{ Sternocleidomastoid } \\
\hline Right & 33.38 & 33.49 & -0.11 & 10 & 4.277 & 0.010 \\
\hline Left & 33.45 & 33.60 & -0.15 & 10 & 3.184 & 0.002 \\
\hline \multicolumn{7}{|l|}{ Masseter } \\
\hline Right & 33.40 & 33.54 & -0.14 & 10 & 7.416 & 0.013 \\
\hline Left & 33.37 & 33.57 & -0.20 & 10 & 3.012 & $<0.001$ \\
\hline \multicolumn{7}{|l|}{ Trapezius } \\
\hline Right & 30.67 & 30.85 & -0.17 & 10 & 6.055 & $<0.001$ \\
\hline Left & 30.73 & 30.93 & -0.20 & 10 & 5.190 & $<0.001$ \\
\hline \multicolumn{7}{|c|}{ Anterior temporalis } \\
\hline Right & 33.59 & 33.79 & -0.20 & 10 & 4.038 & 0.001 \\
\hline Left & 33.35 & 33.49 & -0.14 & 10 & 4.690 & 0.002 \\
\hline
\end{tabular}

A study made by Lurie et al. ${ }^{14}$ demonstrated that $69 \%$ of air force pilots are affected by bruxism and dental occlusion was able to highly influence the air force pilots' muscular system. ${ }^{3}$ Thus an occlusal splint treatment, in which the subject performs an isometric contraction of the mandibular elevator muscles, could be useful for relaxing their masticatory muscles, balancing the occlusal contacts and forces by changes in the rest position due to tooth clenching, which may or may not be related to emotional stress. ${ }^{6,11}$ Moreover, if carefully built, the splint could enhance their postural control, preventing painful lumbar and cervical symptomatologies. ${ }^{2,5,6}$

In this study a temporary occlusal bite was built using Easybite ${ }^{\circledR}$ for analyzing the thermographic effect on air force pilots' facial muscles consequent to the wearing of this splint. Barao et al. ${ }^{7}$ showed that the use of an acrylic splint in a sample of diagnosed myofascial TMD statistically increased the muscles' temperature after approximately 3 mo therapy. These findings corroborate with the results of the present study, since the temperature changed after the Easybite ${ }^{\circledR}$ was worn for $20 \mathrm{~min}$. Notwithstanding, these pilots were not affected by TMD. Their facial muscle tension probably correlated to their bruxism; wearing the temporary splint succeeded in relaxing their muscular system, increasing their facial muscle temperature.

In this study the thermal patterns of the stomatognathic system present a high level of symmetry between the two sides, according to other authors, ${ }^{7,10}$ except for the anterior temporalis that, according to McBeth and Gratt ${ }^{15}$ showed statistical significance between the left and right sides. This may be related to the presence of a parafunction (bruxism), but not the absence of TMD in this sample of air force pilots.

After wearing the occlusal splint, no significant improvement of the temperature asymmetry of the anterior temporalis muscle were recorded, contrary to Barao et al. ${ }^{7}$ Barao at al. found that the mean temperature of the masseter muscle prior to treatment was around $34.2^{\circ} \mathrm{C}$, while in patients with arthritis was significantly lower $\left(33.5^{\circ} \mathrm{C}\right)$. Similar to that, in this study, the simple application of the occlusal splint caused an increase from $33.39^{\circ} \mathrm{C}$ to $33.75^{\circ} \mathrm{C}$ in the masseter muscle.

It should be remembered that, compared to other studies, these results were obtained after only 20 min of application of the occlusal splint. Thus, the significant results of this study are important as a starting point for the planning of a detailed study about the use of an occlusal bite in air force pilots in order to protect their stomatognathic apparatus from bruxism and other stresses and relax their muscles, balancing their occlusal and muscular system.

The principal limitations of this study are the small sample size and the use of a temporary splint for only $20 \mathrm{~min}$. Therefore, additional studies are required to evaluate the muscular temperature changes after prolonged occlusal bite therapy in air force pilots. Within the limitations of this study, the use of an occlusal splint could help in increasing muscle temperatures in air force pilots, with consequent relaxation of their facial muscular system. Bruxist pilots present an asymmetry between the temperatures of the anterior temporalis muscle of the left and right side that cannot be balanced after only 20 min of occlusal splint wearing.

\section{ACKNOWLEDGMENTS}

We thank Dr. G. P. Visser for his help and participation in the final preparation of this manuscript.

Authors and affiliations: Alberto Baldini, DDS, Ph.D., Alessandro Nota, DDS, M.Sc., Clementina Cioffi, DDS, Fabiana Ballanti, DDS, and Paola Cozza, DMD, Department of Orthodontics, University of Rome Tor Vergata, Rome, Italy.

\section{REFERENCES}

1. Baldini A, Cravino G. Dental occlusion and athletic performances. A review of literature. Mondo Ortod. 2011; 36(3):131-141.

2. Baldini A, Nota A, Assi V, Ballanti F, Cozza P. Intersession reliability of a posturo-stabilometric test, using a force platform. J Electromyogr Kinesiol. 2013; 23(6):1474-1479.

3. Baldini A, Nota A, Cravino G, Cioffi C, Rinaldi A, Cozza P. Influence of vision and dental occlusion on body posture in pilots. Aviat Space Environ Med. 2013; 84(8):823-827.

4. Baldini A, Nota A, Cozza P. The association between occlusion time and temporomandibular disorders. J Electromyogr Kinesiol. 2014; pii: S10506411(14)00178-3.

5. Baldini A, Nota A, Tripodi D, Longoni S, Cozza P. Evaluation of the correlation between dental occlusion and posture using a force platform. Clinics (Sao Paulo). 2013; 68(1):45-49. 
6. Baldini A, Tecco S, Cioffi D, Rinaldi A, Longoni S. Gnatho-postural treatment in an air force pilot. Aviat Space Environ Med. 2012; 83(5): 522-526.

7. Barão VA, Gallo AK, Zuim PR, Garcia AR, Assuncão WG. Effect of occlusal splint treatment on the temperature of different muscles in patients with TMD. J Prosthodont Res. 2011; 55(1):19-23.

8. Canavan D, Gratt BM. Electronic thermography for the assessment of mild and moderate temporomandibular joint dysfunction. Oral Surg Oral Med Oral Pathol Oral Radiol Endod. 1995; 79(6):778-786.

9. Costa AC, Dibai Filho AV, Packer AC, Rodrigues-Bigaton D. Intra and inter-rater reliability of infrared image analysis of masticatory and upper trapezius muscles in women with and without temporomandibular disorder. Braz J Phys Ther. 2013; 17(1):24-31.

10. Gratt BM, Sickles EA. Thermographic characterization of the asymptomatic temporomandibular joint. J Orofac Pain. 1993; 7(1):7-14.

11. Hickman DM, Cramer R. The effect of different condylar positions on masticatory muscle electromyographic activity in humans. Oral Surg Oral Med Oral Pathol Oral Radiol Endod. 1998; 85(1):18-23.

12. Johnson JM. Exercise and the cutaneous circulation. Exerc Sport Sci Rev. 1992; 20:59-97.

13. Kenney WL, Johnson JM. Control of skin blood flow during exercise. Med Sci Sports Exerc. 1992; 24(3):303-312.

14. Lurie O, Zadik Y, Einy S, Tarrasch R, Raviv G, Goldstein L. Bruxism in military pilots and non pilots: tooth wear and psychological stress. Aviat Space Environ Med. 2007; 78(2):137-139.

15. McBeth SB, Gratt BM. Thermographic assessment of temporomandibular disorders symptomology during orthodontic treatment. Am J Orthod Dentofacial Orthop. 1996; 109(5):481-488.
16. Merla A, Mattei PA, Di Donato L, Romani GL. Thermal imaging of cutaneous temperature modifications in runners during graded exercise. Ann Biomed Eng. 2010; 38(1):158-163.

17. Merla A, Romani GL. Biomedical applications of functional infrared imaging. Proceedings of the 2005 IEEE Engineering in Medicine and Biology, 27th Annual Conference; September 1-4, 2005; Shanghai, China. New York: IEEE; 2005.

18. Rodrigues-Bigaton D, Dibai-Filho AV, Packer AC, Costa AC, de Castro EM. Accuracy of two forms of infrared image analysis of the masticatory muscles in the diagnosis of myogenous temporomandibular disorder. J Bodyw Mov Ther. 2014; 18(1):49-55.

19. Tecco S, Festa F, Salini V, Epifania E, D'Attilio M. Treatment of joint pain and joint noises associated with a recent TMJ internal derangement: a comparison of an anterior repositioning splint, a full-arch maxillary stabilization splint, and an untreated control group. Cranio. 2004; 22(3):209-219.

20. Tripp LD, Warm JS, Matthews G, Chiu P, Werchan P, Deaton JE. +Gz acceleration loss of consciousness: time course of performance deficits with repeated experience. Hum Factors. 2006; 48(1):109-120.

21. Uematsu S. Thermographic imaging of cutaneous sensory segment in patients with peripheral nerve injury: skin temperature stability between sides of the body. J Neurosurg. 1985; 62(5):716-720.

22. Wieckiewicz M, Paradowska-Stolarz A, Wieckiewicz W. Psychosocial aspects of bruxism: the most paramount factor influencing teeth grinding. Biomed Res Int. 2014; 2014:469187.

23. Zadik Y. Barodontalgia: what have we learned in the past decade? Oral Surg Oral Med Oral Pathol Oral Radiol Endod. 2010; 109(4):e65-e69.

24. Zadik Y. Aviation dentistry: current concepts and practice. Br Dent J. 2009; 206(1):11-16. 